\title{
Molecular evolution and functional characterisation of an ancient phenylalanine ammonia-lyase gene (NnPAL1) from Nelumbo nucifera: novel insight into the evolution of the PAL family in angiosperms
}

Zhihua Wu', Songtao Gui ${ }^{1}$, Shuzhen Wang ${ }^{2}$ and Yi Ding ${ }^{1 *}$

\begin{abstract}
Background: Phenylalanine ammonia-lyase (PAL; E.C.4.3.1.5) is a key enzyme of the phenylpropanoid pathway in plant development, and it catalyses the deamination of phenylalanine to trans-cinnamic acid, leading to the production of secondary metabolites. This enzyme has been identified in many organisms, ranging from prokaryotes to higher plants. Because Nelumbo nucifera is a basal dicot rich in many secondary metabolites, it is a suitable candidate for research on the phenylpropanoid pathway.

Results: Three PAL members, NnPAL1, NnPAL2 and NnPAL3, have been identified in N. nucifera using genome-wide analysis. NnPAL1 contains two introns; however, both NnPAL2 and NnPAL3 have only one intron. Molecular and evolutionary analysis of NnPAL1 confirms that it is an ancient PAL member of the angiosperms and may have a different origin. However, PAL clusters, except NnPAL1, are monophyletic after the split between dicots and monocots. These observations suggest that duplication events remain an important occurrence in the evolution of the PAL gene family. Molecular assays demonstrate that the mRNA of the NnPAL1 gene is 2343 bp in size and encodes a 717 amino acid polypeptide. The optimal pH and temperature of the recombinant NnPAL1 protein are 9.0 and $55^{\circ} \mathrm{C}$, respectively. The NnPAL1 protein retains both PAL and weak TAL catalytic activities with $\mathrm{K}_{\mathrm{m}}$ values of $1.07 \mathrm{mM}$ for L-phenylalanine and $3.43 \mathrm{mM}$ for L-tyrosine, respectively. Cis-elements response to environmental stress are identified and confirmed using real-time PCR for treatments with abscisic acid (ABA), indoleacetic acid (IAA), ultraviolet light, Neurospora crassa (fungi) and drought.

Conclusions: We conclude that the angiosperm PAL genes are not derived from a single gene in an ancestral angiosperm genome; therefore, there may be another ancestral duplication and vertical inheritance from the gymnosperms. The different evolutionary histories for PAL genes in angiosperms suggest different mechanisms of functional regulation. The expression patterns of NnPAL1 in response to stress may be necessary for the survival of N. nucifera since the Cretaceous Period. The discovery and characterisation of the ancient NnPAL1 help to elucidate PAL evolution in angiosperms.
\end{abstract}

Keywords: Nelumbo nucifera, Phenylalanine ammonia-lyase gene, Evolution, Expression, Bioinformatics analysis

\footnotetext{
* Correspondence: yiding@whu.edu.cn

${ }^{1}$ State Key Laboratory of Hybrid Rice, Department of Genetics, College of Life Sciences, Wuhan University, Wuhan, Hubei Province 430072, People's

Republic of China

Full list of author information is available at the end of the article
} 


\section{Background}

The phenylpropanoid pathway is an important branch of the plant secondary metabolism pathways that produces many essential secondary metabolites. In this pathway, secondary metabolic products, such as lignin, flavonoids and coumarins, play important roles in plant growth, development, mechanical support, and disease resistance [1,2]. Phenylalanine ammonia-lyase (PAL; E.C.4.3.1.5) is the first and key enzyme between primary and secondary metabolism, and it catalyses the biotransformation of L-phenylalanine to trans-cinnamic acid. The synthesis of many secondary metabolites, such as flavonoids, flavonols, anthocyanins, condensed tannins, lignins, coumarins, and ubiquinone occur downstream of the phenylpropanoid pathway, [3-6] and is controlled by PAL. Koukol and Conn reported the first plant PAL in 1961. Currently, it is known that the PAL is widely found in all higher plants, a few fungi, and a single prokaryote, Streptomyces, but not animals [7]. Furthermore, PAL shows potential to treat human phenylketonuria, an inborn error of phenylalanine metabolism [8]. Several studies [9-12] have shown that the PALs from Rhodotorula photosynthetic bacteria and monocot plants also utilise tyrosine in addition to phenylalanine; however, the dicot PALs only utilise Phe efficiently. During the past four decades, many PAL genes have been cloned and studied from various plants, such as Ginkgo biloba [13], Ephedra sinica [14], Oryza sativa [15], Isatis indigotica [16], Arabidopsis thaliana [17], Jatropha curcas [18], and Lycoris radiate [19], and the first crystal structure of a plant PAL was determined from parsley (Petroselinum crispum) [20]. PAL exists as a small multigene family, consisting of 2-6 members; however, some species contain additional member, such as potato ( $\sim 40$ copies) [21] and tomato ( 26 copies) [22]. During the evolution of higher plants, the plant PAL genes diversified into various functions in each species, such as Arabidopsis thaliana [23]. Another important ammonia lyase, histidine ammonialyase (HAL), is found in prokaryotes and animals and plays roles in the general histidine degradation pathway. The crystal structure of HAL from Pseudomonas putida revealed its catalytic mechanism of novel polypeptide modification [24]. Despite large differences in the primary sequence of proteins, PAL functions as a tetramer, similar to HAL in vivo. Presumably, PAL developed from HAL when fungi and plants diverged from the other kingdoms [7,25].

Nelumbo nucifera (Nelumbo, Nelumbonaceae) $(2 \mathrm{n}=16)$ is a perennial aquatic plant with ornamental flowers of medicinal and phylogenetic importance. $N$. nucifera produces a series of important secondary metabolites, including alkaloids, flavonoids, steroids, triterpenoids, glycosides and polyphenols [26]. The $N$. nucifera secondary metabolites have a wide range of medical functions and also play important roles in the response to environmental stress, such as pathogen attack and ultraviolet damage. For example, it has been reported that benzylisoquinoline alkaloids and flavonoids from the leaves of $N$. nucifera are a potential candidate for HIV therapy [27]. Nelumbo has survived since the Late Cretaceous, along with a number of other relicts, including Ginkgo, Sequoia, Metasequoia, and Liriodendron [28]. It remains to be determined the mechanism by which PAL evolution has allowed N. nucifera to adapt to harsh environmental stress. Along with the $N$. nucifera genome project $[29,30]$, high-throughput sequencing data will provide a foundation for identifying the key genes in metabolic pathways. However, related research for $N$. nucifera is very limited.

In this study, three intact PAL genes in N. nucifera, NnPAL1, NnPAL2 and NnPAL3 are identified by genomewide analysis. NnPAL1 is an ancient PAL member in angiosperms. The objective of this study is to determine the evolutionary origin, gene structure, function, and expression patterns of this gene under stress conditions.

\section{Results}

Genomic identification and exon/intron structure analysis of the PAL gene family in $\mathrm{N}$. nucifera

Based on whole genome sequences of $N$. nucifera, data mining using 4 Arabidopsis thaliana PAL homologues, AtPAL1, AtPAL2, AtPAL3 and AtPAL4, as queries identify three intact PAL genes, NnPAL1, NnPAL2 and NnPAL3 (Additional file 1: Figure S1). NnPAL1, NnPAL2, and NnPAL3 are located on separate virtual chromosomes, Vchr3, Vchr2 and Vchr7, respectively. According to the position of the introns, these genes are divided into the following three types: phase 0 (introns between codons), phase 2 (introns between the first and the second bases of a codon) and phase 3 (introns between the second and the third bases of a codon). NnPAL1 has two introns of phase 0 , whereas NnPAL2 and NnPAL3 have only one intron of phase 2 (Figure 1). In NnPAL2 and NnPAL3, the exon/ intron borders are within a conserved arginine codon (AG/A). The introns of NnPAL2 and NnPAL3 are separated by two exons. The first exon of NnPAL2 encodes 136 amino acids, whereas the first exon of NnPAL3 encodes 130 amino acids. However, two introns split NnPAL1 into three exons, which code for 363, 179 and 175 amino acids, respectively (Additional file 1: Figure S1). Except for NnPAL1, the phase 2 intron of NnPAL2 and NnPAL3 is conserved, similar to other angiosperms during the evolution of angiosperms [31]. A phase 0 intron in NnPAL1 indicates that NnPAL1 has an evolutionary origin different from NnPAL2 and NnPAL3.

Using BLASTP to search the protein database in NCBI, we found that NnPAL1 is more similar to the PAL genes of gymnosperms (73\% identity to GbPAL, ABU49842.1; 72\% identity to PmPAL, ACS28225.2; and 


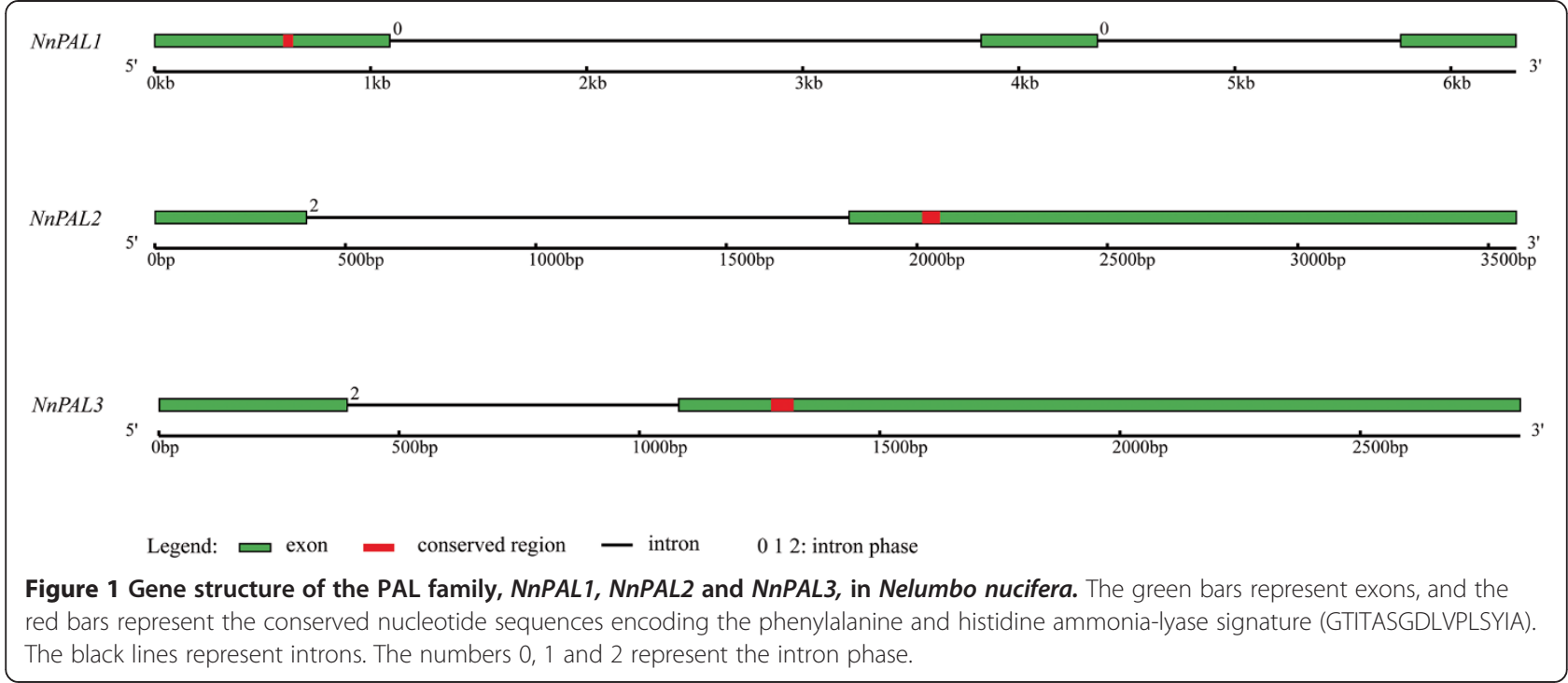

69\% identity to EsPAL, BAG74771.1) than dicots (63\% BnPAL, ABC69916.1; 64\% AtPAL NP_181241.1; and 63\% $D c P A L$, BAC56977.1) (Additional file 2: Figure S2). This is contrary to the phylogeny of $N$. nucifera in the plant kingdom [32]. However, the deduced NnPAL1 protein has the same nine strictly conserved residues, Y112, L140,S204,N260,Q348,Y351,R354,F400, and Q488, that are found in PcPAL of Petroselinum crispum [33]. A typical phenylalanine and histidine ammonia-lyase signature (GTITASGDLVPLSYIA) also exists at position 199-214 (Additional file 3: Figure S3).

\section{Evolutionary analysis of NnPAL1 in N. nucifera}

To understand the evolutionary process of NnPAL1, we use four PAL members, AtPAL1, AtPAL2, AtPAL3 and AtPAL4, from Arabidopsis thaliana to query the Phytozome database. Five monocots and seven dicots that are uniformly distributed in the species tree are selected for analysis (Table 1). Intact PAL amino acids sequences from Pinus taeda are deduced from their transcripts (Additional file 4: Figure S4), and PAL sequences from Physcomitrella patens (Bryophyta) are selected as an outgroup. On the amino acid level, the PAL phylogenetic trees are constructed using the ML (Figure 2), NJ and BI methods (Additional file 5: Figure S5), simultaneously. Five different PALs from Pinus taeda, including Pteda9006, Pteda1143311, Pteda17307, Pteda28316 and Pteda34319, are grouped into three clades as follows: Pteda9006 belongs to Gymnosperm I, Pteda1143311 and Pteda17307 belong to Gymnosperm II, and Pteda28316 and Pteda34319 belong to Gymnosperm III, reported previously [34]. Except for NnPAL1, the other analysed PALs of the dicots and monocots, including NnPAL2 and NnPAL3, are placed in separate monophyletic groups with high bootstrap values of 98 for $\mathrm{ML}$ and 94 for $\mathrm{NJ}$ and a posterior probability value
1.0 for BI (Figure 2 and Additional file 5: Figure S5). As a PAL in N. nucifera, the NnPAL1 gene is clustered together with Pteda1143311 and Pteda17307 (Gymnosperm II) with high bootstrap and high probability values (Figure 2 and Additional file 5: Figure S5). Therefore, the PAL of angiosperms may not be derived from a single paralogue of a gymnosperm PAL. Except for NnPAL1, the PAL clusters from the dicots and monocots are monophyletic after the split between dicots and monocots. This phenomenon suggests that duplication events are an important occurrence during the evolution of the PAL gene family after the split between dicots and monocots [35]. However, the discovery of NnPAL1 indicates that a different evolutionary origin may be responsible for the evolution of the angiosperm PAL genes.

\section{Isolation and bioinformatics characterisation of the full-length NnPAL1 cDNA in N. nucifera}

NnPAL1 has a unique gene structure and phylogenetic position. To determine whether NnPAL1 became a pseudogene during evolution, isolation of the full-length $N n P A L 1$ cDNA is performed from the transcripts of tender leaves. The partial cDNA is obtained by DOP-PCR with degenerate primers. A full-length cDNA containing an open reading frame of $2151 \mathrm{bp}$ is then produced using $5^{\prime}$-RACE and $3^{\prime}$-RACE. Using BLASTN to search the whole genome sequence of $N$. nucifera, we confirm that the newly cloned ancient PAL gene exactly matched the NnPAL1 sequence.

Utilising the ExPASy tool (http://www.expasy.org), the resulting cDNA is determined to encode 717 amino acids with a calculated molecular mass of $77.8 \mathrm{kDa}$ and a theoretical isoelectric point (pI) of 6.64. Additionally, PROSITE (http://prosite.expasy.org/) is used to identify possible posttranslational modification sites, including eight casein kinase II phosphorylation sites, ten protein 
Table 1 Identification of the PAL gene family from the Phytozome database in this study

\begin{tabular}{ll}
\hline Species & PAL seq ID \\
\hline Bryophyta & \\
Physcomitrella patens (10) & Pp1s22_3V6.1 \\
& Pp1s32_311V6.1 \\
Pp1s36_253V6.1 & Pp1s5_197V6.1 \\
Pp1s20_305V6.1 & Pp1s494_3V6.1 \\
Pp1s500_4V6.1 & Pp1s43_88V6.1 \\
Pp1543_67V6.1 & Pp1552_44V6.1
\end{tabular}

Gymnosperm

Pinus taeda (5)

\section{Monocotyledons}

Brachypodium distachyon(6)

Oryza sativa (9)

Sorghum bicolor(6)

Setaria italica (6)

Zea mays (11)

\section{Dicotyledons}

Nelumbo nucifera (3)

Aquilegia coerulea (2)
Pteda1143311

Pteda17307

Pteda9006

Pteda28316

Pteda34319

Bradi5g15830.1

Bradi3g48840.1

Bradi3g47120.1

Bradi3g49260.1

Bradi3g49270.1

Bradi3g49250.2

Os02g41630.2

Os02g41650.1

Os02g41670.1

Os02g41680.1

Os04g43760.1

Os04g43800.1

Os05g35290.1

Os11g48110.1

Os12g33610.1

Sb04g026520.1

Sb04g026560.1

Sb04g026510.1

Sb06g022740.1

Sb06g022750.1

Sb01g014020.1

Si016478m Si016504m Si016467m Si009345m Si009509m Si012256m

GRMZM2G441347_T01 GRMZM2G118345_T01 GRMZM2G447436_T01 GRMZM2G063917_T01 GRMZM2G160541_T01 GRMZM2G081582_T01 GRMZM2G326335_T01 GRMZM2G334660_T01 GRMZM2G170692_T01 GRMZM2G074604_T01 GRMZM2G029048_T01

NnPAL1 NnPAL2 NnPAL3

Aquca_030_00132.1 Aquca_087_00007.1
Table 1 Identification of the PAL gene family from the Phytozome database in this study (Continued)

\begin{tabular}{|c|c|}
\hline Arabidopsis thaliana (4) & $\begin{array}{l}\text { AT2G37040.1(AtPAL1) } \\
\text { AT3G53260.1 (AtPAL2) } \\
\text { AT5G04230.2 } \\
\text { (AtPAL3) } \\
\text { AT3G10340.1 } \\
\text { (AtPAL4) }\end{array}$ \\
\hline Cucumis sativus (8) & $\begin{array}{l}\text { Cucsa.124460.1 } \\
\text { Cucsa.124480.1 } \\
\text { Cucsa.124470.1 } \\
\text { Cucsa.124500.1 } \\
\text { Cucsa.124490.1 } \\
\text { Cucsa.385970.1 } \\
\text { Cucsa.124510.1 } \\
\text { Cucsa.137590.1 }\end{array}$ \\
\hline Glycine $\max (5)$ & $\begin{array}{l}\text { Glyma13g20800.1 } \\
\text { Glyma03g33880.1 } \\
\text { Glyma02g47940.1 } \\
\text { Glyma20g32135.1 } \\
\text { Glyma10g35381.1 }\end{array}$ \\
\hline Mimulus guttatus (3) & $\begin{array}{l}\text { mgv1a001582m } \\
\text { mgv1a019971m } \\
\text { mgv1a002104m }\end{array}$ \\
\hline Populus trichocarpa (4) & $\begin{array}{l}\text { Potri.010G224200.1 } \\
\text { Potri.010G224100.1 } \\
\text { Potri.006G126800.1 } \\
\text { Potri.008G038200.1 }\end{array}$ \\
\hline Vitis vinifera (10) & $\begin{array}{l}\text { GSVIVT01024306001 } \\
\text { GSVIVT01016257001 } \\
\text { GSVIVT01024292001 } \\
\text { GSVIVT01025214001 } \\
\text { GSVIVT01024294001 } \\
\text { GSVIVT01024305001 } \\
\text { GSVIVT01024315001 } \\
\text { GSVIVT01025703001 } \\
\text { GSVIVT01024295001 } \\
\text { GSVIVT01024293001 }\end{array}$ \\
\hline \multicolumn{2}{|c|}{$\begin{array}{l}\text { Note: The PAL gene family identified from one Bryophyta (Physcomitrella } \\
\text { patens), one gymnosperm (Pinus taeda), five monocotyledons (Brachypodium } \\
\text { distachyon, Oryza sativa, Sorghum bicolor, Setaria italica, Zea mays) and eight } \\
\text { dicotyledons (Nelumbo nucifera, Aquilegia caerulea, Arabidopsis thaliana, } \\
\text { Cucumis sativus, Glycine max, Mimulus guttatus, Populus trichocarpa, } \\
\text { Vitis vinifera). }\end{array}$} \\
\hline
\end{tabular}

kinase $\mathrm{C}$ phosphorylation sites, fifteen $\mathrm{N}$-myristoylation sites, three $\mathrm{N}$-glycosylation sites, two tyrosine kinase phosphorylation sites and one cAMP- and cGMP-dependent protein kinase phosphorylation site. The TMHMM Server 2.0 (http://www.cbs.dtu.dk/services/TMHMM-2.0/) is used to show that the deduced NnPAL1 protein is translated and located in the intracellular matrix. The SOPMA tool (http://pbil.ibcp.fr/htm/index.php) is used to predict the secondary structure of the NnPAL1 protein, and indicates that NnPAL1 predominantly consists of alpha helices $(57.32 \%)$ and random coils (30.40\%), along with sheets (7.11\%) and beta turns (5.16\%) (Figure 3A).

Based on the crystal structure of PcPAL (1 W27), the SWISS-MODEL software is used to predict the threedimensional structure of the NnPAL1 protein. The result indicate that NnPAL1 comprises an MIO domain, 


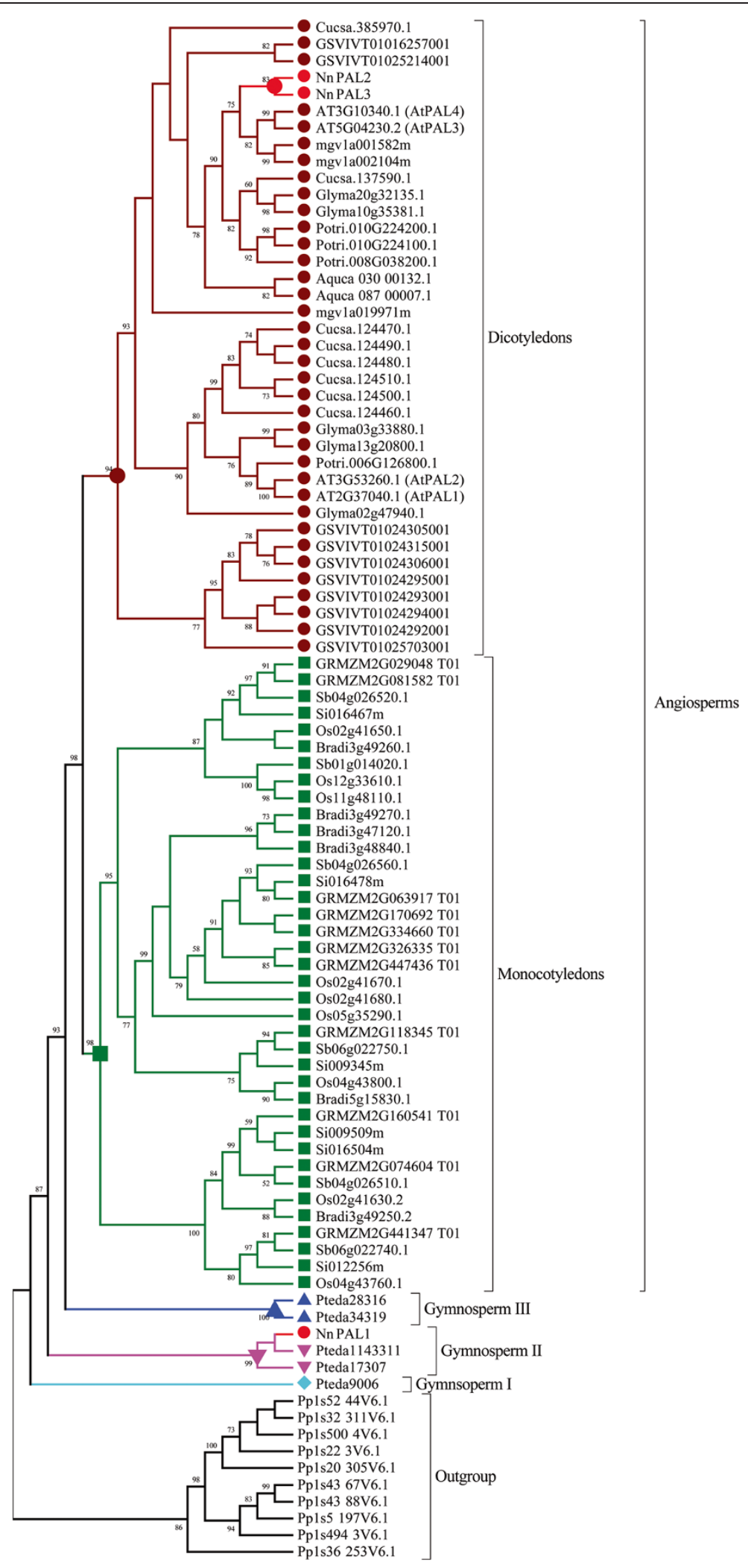

Figure $\mathbf{2}$ (See legend on next page.) 
(See figure on previous page.)

Figure 2 Phylogenetic tree of the phenylalanine ammonia lyase gene family. The amino acid sequences are aligned and the maximum likelihood tree as constructed using the program PhyML 3.0. The numbers at the nodes are the bootstrap values $(>50 \%)$ from the maximum likelihood (ML). The other BI and NJ trees are shown in Additional file 5, Figure 5(A) and Figure 5(B). The numbers associated with the branches are the ML bootstrap support values and posterior probabilities. NnPAL1 is marked with a red dot, and the dicotyledon and monocotyledon clades are marked with carmine and green dots, respectively. Three clades, gymnosperm I, gymnosperm II and gymnosperm III, of Pinus taeda are marked with light green, pink and blue dots, respectively.

core domain and shielding domain [20]. Moreover, a highly conserved Ala-Ser-Gly triad [7] that can be converted autocatalytically is also identified within NnPAL1 (Figure 3B). The results of the bioinformatics prediction and structural analysis of NnPAL1 indicate that NnPAL1 has similar structural features to the reported angiosperm PAL proteins.

\section{Purification and functional characterisation of recombinant NnPAL1}

To confirm the expression of NnPAL1, the recombinant (His) ${ }_{6}$-tagged protein is heterogeneously produced in E. coli BL21 (DE3) and eluted with a series of imidazole buffers (Figure 4B). The size of the expressed and purified recombinant $(\mathrm{His})_{6}$-NnPAL1 protein is confirmed as $\sim 81 \mathrm{kDa}$ by SDS-PAGE (Figure 4A), which is consistent with the predicted mass of NnPAL1 $(\sim 78 \mathrm{kDa})$ combined with a His tag $(\sim 3 \mathrm{kDa})$. Compared to the production at $4 \mathrm{~h}$ and $12 \mathrm{~h}$, the recombinant NnPAL1 $(\sim 81 \mathrm{kDa})$ is expressed maximally at $8 \mathrm{~h}$. The optimal elution concentration of the imidazole buffer is $200 \mathrm{mM}$. The recombinant NnPAL1 protein has both PAL and TAL activities simultaneously, although phenylalanine ammonia-lyase from dicots only utilises Phe efficiently [33]. A study of the physicochemical properties shows that its optimal $\mathrm{pH}$ and temperature are $\mathrm{pH} 9.0$ and $55^{\circ} \mathrm{C}$, respectively. The NnPAL1 $K_{m}$ values for L-phenylalanine and L-tyrosine are $1.07 \mathrm{mM}$ and $3.43 \mathrm{mM}$, respectively.

\section{Expression profile of NnPAL1 under stress conditions}

Because of the accumulation important secondary metabolites, such as alkaloids and flavonoids, these phenylpropanoid compounds from $N$. nucifera leaves play essential roles in stress resistance. PAL is vital to the phenylpropanoid pathway that leads to the production of these secondary metabolites. The upstream cis-elements of NnPAL1 (Additional file 1: Figure S1), including the related regulatory elements, such as the MYB binding

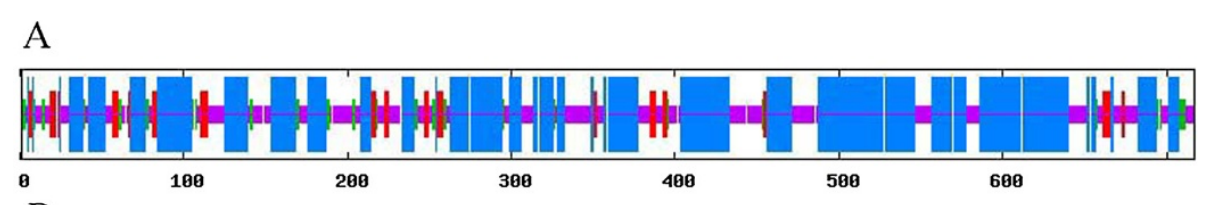

B

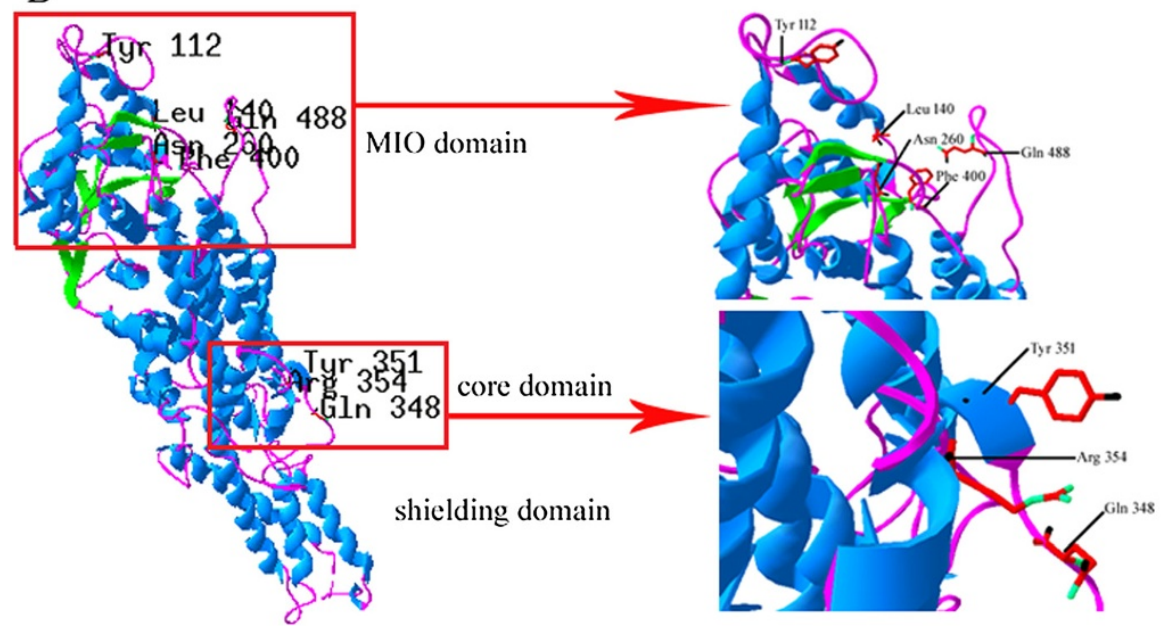

Figure 3 Prediction of NnPAL1 secondary structure and tertiary structure. (A) Prediction of the NnPAL1 secondary structure. The blue, pink, red, and green regions represent the alpha helix, random coil, extended strand, and beta turn, respectively. (B) The three domains of the predicted tertiary structure of NnPAL1 established by homology-based modelling ( 9 strictly conserved residues are marked). 


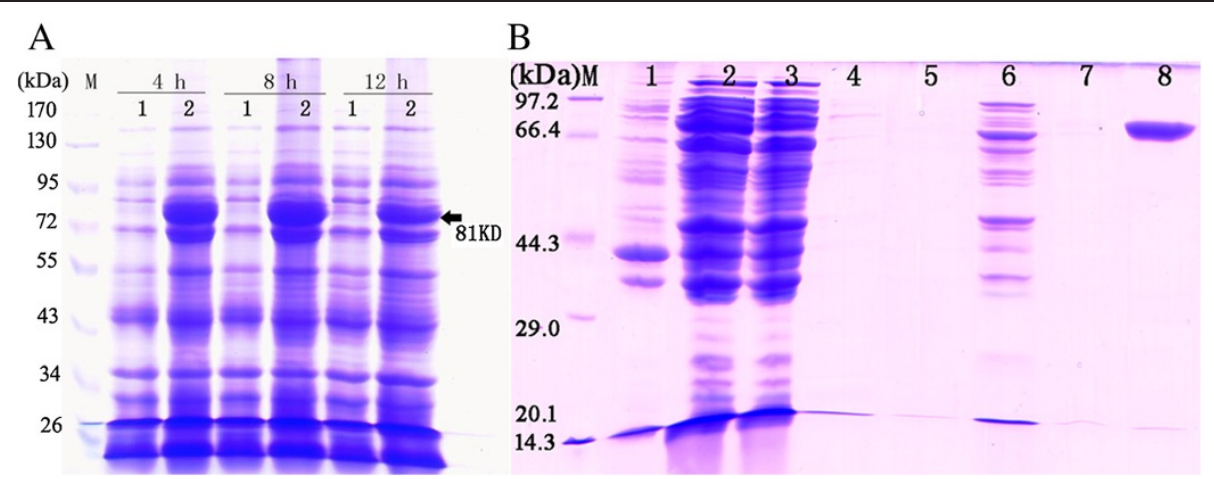

Figure 4 Expression (A) and purification (B) of recombinant NnPAL1. A: The total proteins from E. coli BL21 are harvested at $4 \mathrm{~h}, 8 \mathrm{~h}$ and $12 \mathrm{~h}$ after post-induction, and 1 and 2 represent the total proteins of $E$.coli BL21 harbouring the pET28a(+) vector and recombinant pET28a (+)-NnPAL1 vector, respectively. B: A series of imidazole buffer concentration gradients (10 mM, 50 mM, 100 mM, 200 mM); lane1: the supernatant of the E. coli BL21 lysate harbouring the pET28a(+) vector; lane2: (native control) the supernatant of the E. coli BL21 lysate harbouring the pET28a (+)-NnPAL1 vector; lane3: the supernatant of the flow through of the Ni-IDA column for three replicates; lane 4, lane 5, lane 6, lane 7 and lane 8: the products washed with $10 \mathrm{mM}, 20 \mathrm{mM}, 50 \mathrm{mM}, 100 \mathrm{mM}$, and $200 \mathrm{mM}$ imidazole buffer, respectively.

site involved in drought-inducibility (CAACTG), auxinresponsive element (AACGAC), fungal elicitor responsive element (TTGACC), cis-acting element involved in abscisic acid responsiveness (CACGTG), and light responsive element (CACGTG, CACGAC, CACGTG) are identified. Under different stress conditions, including ABA ( $250 \mu \mathrm{M}$ abscisic acid), IAA (100 ng/ml), ultraviolet light, Neurospora crassa (fungi) and drought, the expression of NnPAL1 is induced in N. nucifera leaves (Figure 5A-E). After 4 hours, PAL expression is maximal with ABA, IAA, ultraviolet light and Neurospora crassa (fungi), and after 8 hours, PAL expression is maximal with drought treatment. We conclude that these corresponding elements perform an important role in the response to internal and external environmental stimulus.

\section{Discussion}

Identification of the PAL family in N. nucifera from whole genomic sequences

The genomic DNA used for de novo sequencing is extracted from the clean shoots of $N$. nucifera. We use sixteen assembled virtual chromosomes of high quality as the resource for the PAL search. In previous reports of PAL from higher plants, all functionally identified PAL genes [13-23] encode approximately 700 amino acids and contain the characteristic conserved GTITASGDLVPLSYIA motif. Therefore, we set the sizes of the PAL family to larger than 500 amino acids with the GTITASGDLVPLSYIA signature. Three PAL genes, NnPAL1, NnPAL2 and NnPAL3, are located in the well-defined regions of the assembled sequences. Consistent with the phylogeny of angiosperms, NnPAL2 and NnPAL3 are similar to the PAL from dicots. However, NnPAL1 is similar to the PAL from gymnosperms. The full-length cDNA of NnPAL1 is cloned from the RNA transcripts of tender leaves using RACE method. NnPAL1 is transcribed with an intact open reading frame, suggesting that it does not become a pseudogene during evolution.

\section{NnPAL1 from the genuine PAL family of N. nucifera}

$N$. nucifera is a perennial aquatic plant. Therefore, obtaining pure tissues is a prerequisite for molecular biology experiments. An endophyte is a bacterial or fungal microorganism, which colonises inter- and/or intracellularly inside the healthy tissues of the host plant [36]. We are careful to remove the residues from both shoots and leaves. To confirm that NnPAL1 is a member of the PAL family of N. nucifera and not endophytes, we performed several experiments.

First, we determine the location of NnPAL1 in Vchr3 and extract the upstream sequence $(31,942 \mathrm{bps})$ and downstream sequence $(26,288$ bps) flanking NnPAL1 (Additional file 6). Then, we performed a discontiguous megablast search against the nucleotide collection database in NCBI to search for homologous regions. In the upstream 1-5000 bps of NnPAL1, we find out a highly homologous region to dicots. The sequences with the first three highest scores are uncharacterised mRNA from Vitis vinifera, the mRNA of a tetratricopeptide repeatcontaining family protein in Populus trichocarpa, and an mRNA of a conserved hypothetical protein in Ricinus communis. In the downstream 10,000-15,000 bps of NnPAL1, we find out a homologous partial coding sequence of the GWD gene for alpha-glucan water dikinase from $N$. nucifera. A homologous region to dicots is also identified in the downstream 20,000-25,000 bps. The sequences with the first three highest scores are the mRNA of a zinc finger $\mathrm{CCCH}$ domain-containing protein 17-like in Citrus sinensis and the mRNA of a zinc finger family protein in Populus trichocarpa (Additional file 7). As a basal dicot, the flanking sequences of $N$. nucifera NnPAL1 show homology to sequences of all the other dicots. 


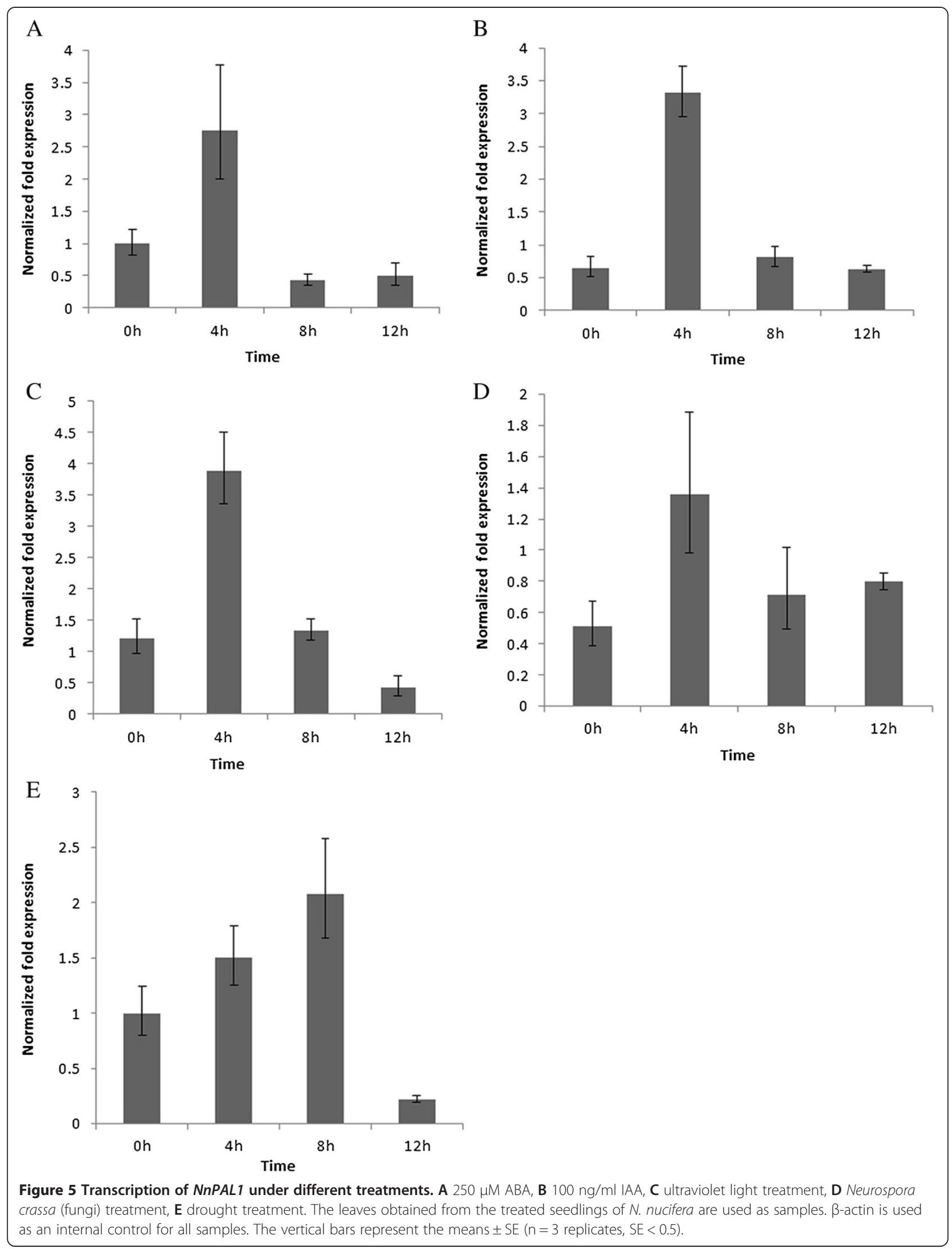


Second, the identities between plant PALs and HALs and the PALs of microorganisms and animals are compared. Because PAL has the same catalytic mechanism as HAL, it is hypothesised to have developed from HAL when fungi and plants diverged from the other kingdoms. HAL is widely distributed among prokaryotes and animals. We extracted the prokaryote and animal HALs, and only one prokaryote, Streptomyces, and fungal PAL from NCBI the database are identified in addition to the plant PAL from our study. The identities of plant PAL to the prokaryote and animal HAL, Streptomyces PAL, fungal PAL, and plant PAL are approximately 18\%, 18\%, $25 \%$ and $64 \%$, respectively (Additional file 8 ). The conclusion that there are significant sequence difference among these PALs and HALs is consistent with a previous report [20]. Similar to the other plant PALs, the sequence of NnPAL1 is much more similar to other plant PALs, and distant from the PALs and HALs of microorganisms. Phylogenetic analysis of HAL and PAL using the neighbour-joining method demonstrates that they form three separate clades, prokaryote and animal HAL, including Streptomyces PAL, fungal PAL, and plant PAL (Figure 6). Therefore, NnPAL1 is not from the endophytes. Based on these results, we can infer that NnPAL1 is a genuine member of the PAL family from $N$. nucifera, but not endophytes.

\section{Evolution of NnPAL1 in N. nucifera during the evolution of plants}

In this study, three PALs, NnPAL1, NnPAL2 and NnPAL3, are identified using the database of whole genomic sequences as a resource. In previous reports, the angiosperm PAL had phase 2 introns at an Arg codon of [15,31,37,38], but the gymnosperm PAL had no intron [13]. Similarly, both NnPAL2 and NnPAL3 have only one intron of phase 2 , whereas NnPAL1 has two introns of phase 0 . This result demonstrates that $N n P A L 1$ has unique gene structure that is different from NnPAL2, NnPAL3 and other PAL genes from angiosperms. This difference between $N n P A L 1$ and other angiosperm PALs suggests that it is an ancient gene with a different evolutionary origin.

PAL and HAL are members of the lyase class I_like superfamily of enzymes, which catalyse similar betaelimination reactions and are active as homotetramers. PAL and HAL diverged from each other when fungi and plants diverged from the other kingdoms (Figure 6). Because of their similar structures, PAL is derived from the His ammonia-lyase. HAL is a basic enzyme, participating in a central metabolic pathway, and PAL is derived from HAL to fulfil specific functions.

PAL is a ubiquitous higher-plant enzyme that catalyses the nonoxidative deamination of phenylalanine to transcinnamic acid. However, the origin and evolution of the PAL gene family in seed plants (Spermatophyta) have not been determined [31,39]. Currently, two major mechanisms are responsible for the evolution and functional divergence of genes. One evolutionary mechanism is called HGT (horizontal gene transfer) and refers to the movement of genes between different species [40]. HGT events occur only in plant mitochondrial genes [41-43], and rarely in nuclear genes [44]. The other evolutionary mechanism is gene duplication, which is the main mechanism for evolutionary innovations and functional divergence [45]. Based on morphological characteristics and molecular data, gymnosperms are considered ancestral to the angiosperms [39]. At least three ancestral duplication events of PAL occurred, leading to three clades of gymnosperm PAL genes, gymnosperm-I, gymnosperm-II and gymnosperm-III. It appears that angiosperms diverged from gymnosperm III when only one paralogue PAL gene is retained within the angiosperms [31]. In this study, we construct PAL phylogenetic trees that include the PAL gene families from Pinus taeda (gymnosperm I, II, III), monocots and dicots according to the gene sequences of the sequenced species (Figure 2 and Additional file 5: Figure S5). The phylogenetic trees show that NnPAL1 is clustered together with Pteda1143311 and Pteda17307 (gymnosperm II); however, NnPAL2 and NnPAL3 are clustered with dicots with high bootstrap and posterior probability values. Perhaps, NnPAL1 has a different evolutionary origin from NnPAL2 and NnPAL3. Except for NnPAL1, the other PAL clusters are monophyletic after the split between dicots and monocots (Figure 2 and Additional file 5: Figure S5). However, the PAL from one species is clustered together with the other species rather than with a single species. This result indicates that duplication events are important in the evolution of PAL genes after the split between dicots and monocots $[46,47]$.

During evolution, NnPAL1 is found to be an ancient member of the PAL family that has been retained in angiosperms. A different evolutionary history for PAL genes in angiosperms suggests different mechanisms of functional regulation. In the phylogenetic trees of PAL (Figure 2), NnPAL1 is not found where expected. Interestingly, NnPAL1 shows high homology to Pteda1143311 and Pteda17307 from Pinus taeda, and Pinus taeda is also rich in various secondary metabolites. There may be a shared secondary metabolite produced by NnPAL1 or Pteda1143311 and Pteda17307. Moreover, this specific product may protect $N$. nucifera and Pinus taeda from similarly extreme environments. NnPAL1 may have been essential for $N$.nucifera to survive in harsh environments during the Cretaceous period.

We speculate that the angiosperm PAL is not of monophyletic origin. Ancestral gene duplication and vertical inheritance from gymnosperms may occur during evolution from parent to offspring. In gymnosperms another paralogue of the ancient PAL exists that is retained 


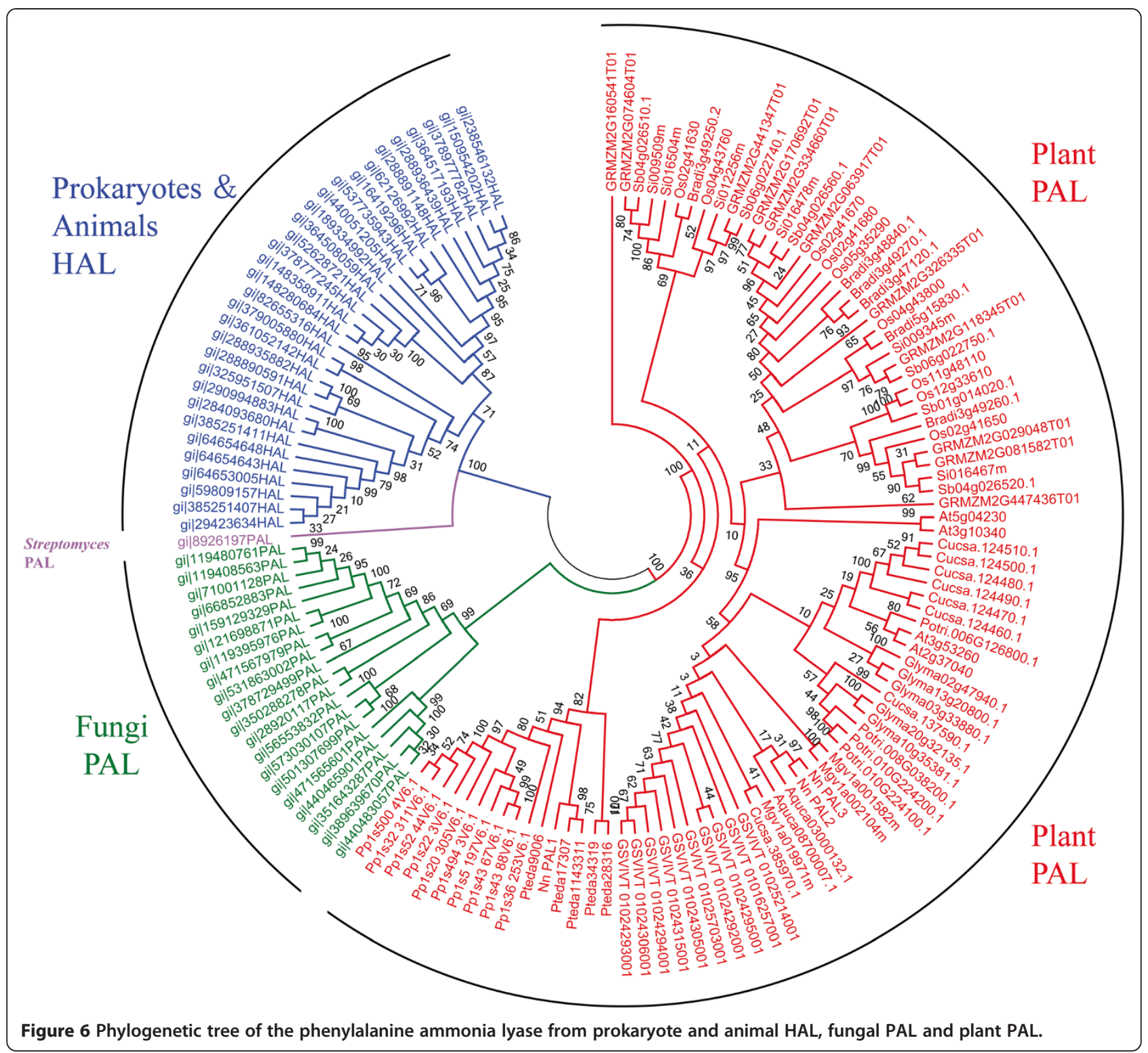

prior to the formation of angiosperms. NnPAL1 may be derived from the product of gymnosperm-II PAL. Discovering a functional NnPAL1 indicates that angiosperm PAL genes are not derived from a single gene in the ancestral angiosperm genome. However, similar modification sites and structure to other angiosperm PALs suggest that NnPAL1 can catalyse the deamination of phenylalanine to trans-cinnamic acid and is involved in the phenylpropanoid pathway.

\section{Functional characterisation and expression patterns of NnPAL1}

During evolution, NnPAL1 remained functional with both PAL and TAL activities. Compared with other PALs cloned from other plants $[14,18]$, both the PAL and TAL activities of NnPAL1 show higher $\mathrm{K}_{\mathrm{m}}$ values, which can be explained as follows: (I) during the evolution of angiosperms, the function of the most ancient PAL (NnPAL1) is gradually replaced by a new PAL; (II) NnPAL1 has many posttranslational modification sites, which may be involved in the subunit turnover of NnPAL1 in vivo [48], and prokaryote expression systems lack multiple protein modifications, which affect enzyme protein stability; and (III) the ancient NnPAL1 has evolved a novel function required for other metabolic pathways [49]. The optimum $\mathrm{pH}$ is 9.0 and the optimum temperature is $55^{\circ} \mathrm{C}$, which is similar to other PALs from higher plants [18]. The expression patterns are validated by real-time PCR. In response to environmental stress during the Cretaceous period, $N$. nucifera is eventually trapped in aquatic areas of Asia [50]. 
This type of environment makes secondary metabolites important for $N$. nucifera because they protect the species from various stimuli. PAL expression is regulated by various factors at the transcriptional level. Bioinformatics analysis of the upstream cis-elements in NnPAL1 identified several related regulatory elements, such as the MYB binding site involved in drought-inducibility, the auxinresponsive element (IAA), the fungal elicitor responsive element, the cis-acting elements involved in abscisic acid responsiveness $(\mathrm{ABA})$, and the light responsive element in NnPAL1 gene. All the treatments used in this study cause increases of the NnPAL1 transcripts, which suggests that NnPAL1 is regulated by these elements. The ancient NnPAL1 of $N$. nucifera is involved in the response to stressful environments, which makes $N$. nucifera the representative of plants that survived from the Cretaceous period [28].

\section{Conclusions}

Using comparative genomics and phylogenetic analyses, three PAL members, NnPAL1, NnPAL2 and NnPAL3, are identified. The distinction between NnPAL1 and other angiosperm PALs suggests that NnPAL1 is not derived from a PAL paralogue of a gymnosperm leading to angiosperms. We postulate that there may be another ancestral duplication event and vertical inheritance from the gymnosperms. The ancient PAL NnPAL1 from N. nucifera is characterised at both the RNA and protein levels in vitro. The unique biochemical characteristics of $N$. nucifera may allow it to overcome the harsh environment. Additionally, as a basal dicot, $N$. nucifera is a perennial aquatic plant with agricultural, evolutionary and medicinal importance $[26,27]$. Polyphenolic compounds in N. nucifera have important pharmacological and physiological activities. The discovery and characterisation of an ancient NnPAL1 provides new insight into PAL evolution in angiosperms and may also lead to improved function through the genetic engineering of $N$. nucifera.

\section{Methods}

\section{Identification of the PAL gene family in N. nucifera}

High purity DNA is extracted from clean and tender shoots of $N$. nucifera, and is used for de novo sequencing. For the de novo assembly, 16.4 Gb of filtered data with 15 -fold depth is used. Sixteen virtual chromosomes $(2 \mathrm{n}=16)$ are assembled with high quality.

We search the orthologues in $N$. nucifera against the whole genome using four Arabidopsis PAL homologs, AtPAL1, AtPAL2, AtPAL3, and AtPAL4. The search criteria are as follows:

1) A local database with the sequences of sixteen virtual chromosomes are constructed on the Bio-Linux platform;
2) tBLASTN is conducted with a cut-off $E$ value of $1 \mathrm{e}-20$ in the local database with each member of the AtPAL family;

3) the aligned frames containing a highly conserved phenylalanine and histidine ammonia-lyase signature (GTITASGDLVPLSYIA) are selected for further analysis;

4) the related genome sequences with intact open reading frames are located in the well-defined region of assembled sequences and are extracted;

5) the extracted codes larger than 500 amino acids are selected and annotated.

\section{Identification of gene families in other plants and construction of the PAL phylogenetic tree}

Based on a conserved phenylalanine and histidine ammonialyase signature, the PAL families from other plants except for Pinus taeda are identified and downloaded from Phytozome (http://www.phytozome.net) with a cutoff $\mathrm{E}$ value of $1 \mathrm{e}^{-20}$. The PAL proteins from Pinus taeda are deduced from their RNA transcripts [34]. The analysed species (Table 1) are as follows: one Bryophyta (Physcomitrella patens), one gymnosperm (Pinus taeda), five monocotyledons (Brachypodium distachyon, Oryza sativa, Sorghum bicolor, Setaria italica, Zea mays), and eight dicots (Aquilegia caerulea, Arabidopsis thaliana, Cucumis sativus, Glycine max, Mimulus guttatus, Populus trichocarpa, Vitis vinifera, and Nelumbo nucifera). The protein sequences are aligned with the CLUSTALW program [51] with manual adjustments. The phylogenetic trees are simultaneously inferred from the protein alignment using three methods as follows: the NJ (Neighbour-joining) tree with the JTT model, the ML (maximum likelihood) tree with LG model, and BI (Bayesian inference) tree with the GTR model, are generated with Mega 5 [52], PhyML 3.0 [53] and Mrbayes 3.2 [54], respectively. The bootstrap values are set 1000 for the neighbour-joining and maximum likelihood tree. For Bayesian inference, we sample every 10 generations for 300,000 total generations on two independent parallel runs of the Monte Carlo Markov Chains. Then, the average standard deviation of the split frequencies is calculated to check the convergence of the two runs.

\section{Plant material, cloning and expression vectors}

$N$. nucifera mature seeds are harvested from East Lake of Wuhan, China. The tender leaves are collected when the seedling germinated from seeds in the greenhouse. E.coli top10 (TaKaRa, Dalian, China) is used as the host for plasmid pMD18-T vector (TaKaRa, Dalian, China) amplification. E.coli BL21(DE3) is selected as the host for $\mathrm{pET}-28 \mathrm{a}(+)$ expression vector.

\section{Isolation of the full-length NnPAL1 CDNA}

The total RNA is isolated from the leaves using a modified CTAB method [55]. The first strand CDNA is 
produced by RT-PCR using reverse transcriptase (MBI Fermentas). Two degenerate primers, (Nf-F) 5'-GCNTC NGGHGAYYTDGTBCC-3' and (Nf-R) 5' -ARNCCBA RDGART TWACATC-3', are designed according to the highly conserved regions of the plant PAL for both the amino acid and nucleotide sequences. The partial cDNA of PAL is amplified using the following conditions: initial denaturing at $94^{\circ} \mathrm{C}$ for $4 \mathrm{~min}$, followed by 35 cycles of $94^{\circ} \mathrm{C}$ for $40 \mathrm{~s}, 59^{\circ} \mathrm{C}$ for $40 \mathrm{~s}$, and $72^{\circ} \mathrm{C}$ for $80 \mathrm{~s}$, with a final extension at $72^{\circ} \mathrm{C}$ for $10 \mathrm{~min}$. The target fragment is checked on $1 \%$ agarose gel and purified with a Gel Extraction Kit (BioDev-Tech, Beijing, China). The purified products are then ligated into the pMD18-T Easy vector (TaKaRa, Dalian, China), transformed into competent $E$. coli Top10 and sequenced on an ABI 3730.

The full-length cDNA of the PAL gene is isolated by 3 ' - and 5 '-RACE using the RACE Kit (TaKaRa, Dalian, China). Based on the sequenced DNA fragment, four gene-specific primers, 3'GSP1 (5'-CTGGACTACGGAT TCAAGGGTG-3'), 3'GSP2 (5'-TCAGTATTTGGCAA ACC CAGTCA-3'), 5'GSP1(5' -AGCATCACTTCGCA GAACATCG-3') and 5'GSP2 (5'-GTACGGAC CTTGG AGTTGGGAC-3'), are designed for the $3^{\prime}$ - and $5^{\prime}$ RACE experiments, respectively. A 860-bp fragment and 791-bp fragment are then obtained by $3{ }^{\prime}$-RACE and 5 'RACE, respectively. The full-length coding CDNA of the 2154-bps is amplified and sequenced using two genespecific primers, 5'-GAATTCATGGTTGCAGGGGCC GAGATAG-3' and 5' -CCCTCGAGCACAAGAAGGCA ACACCAAAGT-3'.

\section{Bioinformatics analysis of NnPAL1}

The amino acid sequence and protein analysis of NnPAL1 are performed with the ExPASy tools (http://us. expasy.org/tools) and NCBI server (http://www.ncbi. nlm.nih.gov/). The possible posttranslational modification sites are predicted by PROSITE (http://prosite. expasy.org/). The prediction of secondary structure and trans-membrane helices in the PAL protein are performed with SOPMA (http://pbil.ibcp.fr/htm/index.php) and the TMHMM Server v. 2.0 (http://www.cbs.dtu.dk/ services/TMHMM-2.0/), respectively. Homology modelling is performed with Swiss-Model (http://swissmodel. expasy.org/) and is based on the PAL crystal structure from Petroselinum crispum [20].

\section{Expression of NnPAL1 in E. coli}

Primers NnP1 (5'-GAATTCATGGTTGCAGGGGCCG AGATAG-3', the italics is EcoRI restriction site) and $\mathrm{NnP2}$ (5'-CCCTCGAGCACAAGAAGGCAACACCAA AGT-3', the italics is $X h o I$ restriction site) are used to amplify the NnPAL1 gene coding sequence. The PCR products are digested with EcoRI and $X h o I$ and then inserted into pET28a(+) expression vector. The recombinant plasmid
NnPAL1-pET28a(+) is transformed into the BL21 strain and sequenced to confirm the correct ORF of NnPAL1.

The transformant with the correct NnPAL1-pET28a $(+)$ is selected and cultured in Luria-Bertani (LB) medium containing $50 \mu \mathrm{g} / \mathrm{ml}$ kanamycin at $37^{\circ} \mathrm{C}$ until the $\mathrm{OD}_{600}$ reached 0.6. Protein expression is induced with $0.5 \mathrm{mM}$ isopropyl $\beta$-D-1-thiogalactopyranoside (IPTG) at $16^{\circ} \mathrm{C}$ for $12 \mathrm{~h}$. The recombinant proteins are purified on a Ni-NTA agarose column and eluted with a step gradient of imidazole buffers $(10 \mathrm{mM}, 50 \mathrm{mM}$, $100 \mathrm{mM}$, and $200 \mathrm{mM}$ ). The purity of the recombinant protein is verified by SDS-PAGE. The $200 \mathrm{mM}$ fractions are dialysed with Spectra/Por Membranes (MWCO: $8,000-14,000)$ in dialysis buffer.

Enzyme activity assay for the recombinant NnPAL1 protein The protein concentrations are determined with the G250 dye-binding method [56] using bovine serum albumin as the protein standard. The enzyme activity of the recombinant NnPAL1 is assayed by measuring the transcinnamic acid formation at $290 \mathrm{~nm}$ [57] and p-coumaric acid formation at $310 \mathrm{~nm}$ [9]. The PAL activity and TAL activity is expressed in nkat (nanomole of trans-cinnamic acid/p-coumaric acid formed per second).

To determine the optimum temperature and optimum $\mathrm{pH}$ for enzyme activity, several assays are performed at $\mathrm{pH} 8.5$ for $30 \mathrm{~min}$ at varying temperatures $(4,23,30,37$, $45,50,55,60,70,80$ and $90^{\circ} \mathrm{C}$ ), and at $37^{\circ} \mathrm{C}$ for $30 \mathrm{~min}$ with buffer of various of $\mathrm{pH}(5,6,7,7.5,8,8.5,9,10$, $11)$, respectively. The reactions are performed in $150 \mu \mathrm{l}$ reaction mixtures with $6 \mu \mathrm{g}$ recombinant NnPAL1, $15 \mathrm{mM}$ L-phenylalanine and $50 \mathrm{mM}$ Tris- $\mathrm{HCl}(\mathrm{pH} 8.5)$, and are terminated with the addition of concentrated $\mathrm{HCl}$ [57].

To determine the kinetic parameters and substrate specificity, $150 \mu \mathrm{l}$ reaction mixtures containing $6 \mu \mathrm{g}$ recombinant NnPAL1 proteins, $50 \mathrm{mM}$ Tris- $\mathrm{HCl}(\mathrm{pH} 8.5)$ and a range of L-phenylalanine $(0.15-15 \mathrm{mM})$ or L-tyrosine (0.3-2 mM) concentrations are used. Hyperbolic plots and double reciprocal plots (Lineweaver-Burk plot) are used to calculate the $\mathrm{K}_{\mathrm{m}}$ (Michaelis-Menten constant) using the Michaelis-Menten equation [35].

\section{Cis-regulatory element analysis and expression of NnPAL1 by quantitative real-time PCR}

The 5' upstream region of NnPAL1 is characterised using BLASTN against the whole genomic sequence of $N$. nucifera with NnPAL1 gene. We predicted the cis-elements by submitting 5 ' fragment to PlantCARE (http://bioinformatics.psb.ugent.be/webtools/plantcare/html/).

Two-week-old leaves are treated with $250 \mu \mathrm{M}$ abscisic acid (ABA), $100 \mathrm{ng} / \mathrm{ml}$ IAA, ultraviolet light, Neurospora crassa (fungi) and drought according to the cis-elements. The treated leaves are harvested and immediately frozen 
after $0,4,8$, and $12 \mathrm{~h}$. The total RNA is isolated from the leaves and treated with RNase-free DNase I to avoid DNA contamination. Real time RT-PCR analysis of NnPAL1 is performed on an Applied Biosystems StepOne Plus, using $\beta$-actin gene ( $\beta$-actin-F: 5'-CCTGATGGGCAAGTGA TT-3', $\beta$-actin-R: 5'-GCTCATACGGTCAG CAATA-3') as an internal control for all the samples.

\section{Additional files}

Additional file 1: Figure S1. Nucleotide sequences of NnPAL1, NnPAL2 and NnPAL3, upstream cis-elements of NnPAL1 identified from the whole genome sequences of Nelumbo nucifera.

Additional file 2: Figure S2. Sequences alignment of NnPAL1 and other typical PALs from seed plants. The phenylalanine and histidine ammonia-lyase signature (GTITASGDLVPLSYIA) is underlined with red lines, and the conserved Ala-Ser-Gly triad is framed in a red box.

Additional file 3: Figure S3. The nucleotide sequence and deduced amino acid sequence of NnPAL1. The start codon (ATG) and stop codon (TAA) are underlined. The typical phenylalanine and histidine ammonia-lyase signature is boxed. Nine strictly conserved residues, Y112,L140,S204,N260,Q348, Y351,R354,F400,Q488, are marked in red italics.

Additional file 4: Figure S4. Deduced amino acid sequences from the PALs of Pinus taeda.

Additional file 5: Figure S5. Phylogenetic trees of the phenylalanine ammonia lyase gene family constructed using the Bl method (a) and NJ method (b). The posterior probability and bootstrap values (>50\%) for the two trees are shown on each branch, respectively.

Additional file 6: Identification of the upstream sequence (31,942 bps) and downstream sequence (26,288 bps) of NnPAL1.

Additional file 7: Homology search for the upstream sequence ( $31,942 \mathrm{bps})$ and downstream sequence $(26,288 \mathrm{bps})$ against the nucleotide collection database in NCBI.

Additional file 8: Identities of the plant PALs to prokaryote and animal HAL, Streptomyces PAL, fungal PAL and plant PAL, marked with yellow, green, purple and blue, respectively.

\section{Abbreviations}

HAL: Histidine ammonia-lyase; PAL: Phenylalanine ammonia-lyase; TAL: Tyrosine ammonia-lyase; NnPAL 1: One ancient member from PAL gene family of Nelumbo nucifera; DOP-PCR: Degenerate oligonucleotide primer PCR; RACE: Rapid amplification of CDNA ends; ML: Maximum-likelihood; NJ: Neighbor-joining; Bl: Bayesian inference.

\section{Competing interests}

The authors declare that they have no competing interests.

\section{Authors' contributions}

YD and ZHW designed the general idea and experiments of the study. ZHW characterized the sequences, carried out most of the experiments, and drafted the manuscript. STG participated biochemical assays of protein. SZW performed the expression analyses. All authors read and approved the final manuscript.

\section{Acknowledgements}

The authors thank Dr Huabin Zhao in Wuhan University for helps in evolutionary analyses. This research is financially supported by National Natural Science Foundation of China (31271310).

\section{Author details}

${ }^{1}$ State Key Laboratory of Hybrid Rice, Department of Genetics, College of Life Sciences, Wuhan University, Wuhan, Hubei Province 430072, People's Republic of China. ${ }^{2}$ College of Life Sciences, Huanggang Normal University, Huanggang, Hubei Province 438000, People's Republic of China.
Received: 24 March 2014 Accepted: 28 April 2014

Published: 9 May 2014

\section{References}

1. Dixon RA, Paiva NL: Stress-induced phenylpropanoid metabolism. Plant Cell 1995, 7:1085-1097.

2. Pellegrini L, Rohfritsch O, Fritig B, Legrand M: Phenylalanine ammonia-lyase in tobacco: molecular cloning and gene expression during the hypersensitive reaction to tobacco mosaic virus and the response to a fungal elicitor. Plant Physiol 1994, 106:877-886.

3. Hamberger B, Ellis M, Friedmann M, de Azevedo SC, Barbazuk B, Douglas CJ: Genome-wide analyses of phenylpropanoid-related genes in Populus trichocarpa, Arabidopsis thaliana, and Oryza sativa: the Populus lignin toolbox and conservation and diversification of angiosperm gene families. Can J Bot 2007, 85(12):1182-1201.

4. Naoumkina MA, Zhao Q, Gallego-Giraldo L, Dai X, Zhao PX, Dixon RA: Genome-wide analysis of phenylpropanoid defence pathways. Mol Plant Pathol 2010, 11(6):829-846.

5. Raes J, Rohde A, Christensen JH, Van de Peer Y, Boerjan W: Genome-wide characterization of the lignification toolbox in Arabidopsis. Plant Physiol 2003, 133(3):1051-1071.

6. Tsai CJ, Harding SA, Tschaplinski TJ, Lindroth RL, Yuan Y: Genome-wide analysis of the structural genes regulating defense phenylpropanoid metabolism in Populus. New Phytol 2006, 172(1):47-62.

7. MacDonald MJ, D'Cunha GB: A modern view of phenylalanine ammonia lyase. Biochem Cell Biol 2007, 85:273-282.

8. Kim W, Erlandsen H, Surendran S, Stevens RC, Gamez A, Michols-Matalon K, Tyring SK, Matalon R: Trends in enzyme therapy forphenylketonuria. Mol Ther 2004, 10:220-224.

9. Kyndt JA, Meyer TE, Cusanovich MA, Van Beeumen JJ: Characterization of a bacterial tyrosine ammonia lyase, a biosynthetic enzyme for the photoactive yellow protein. FEBS Lett 2002, 512:240-244.

10. Moffitt MC, Louie GV, Bowman ME, Pence J, Noel JP, Moore BS: Discovery of two cyanobacterial phenylalanine ammonia lyases: kinetic and structural characterization. Biochemistry 2007, 46:1004-1012.

11. Ogata K, Uchiyama K, Yamada H: Metabolism of aromatic amino acid in microorganisms. Part I: formation of cinnamic acid from phenylalanine. Agric Biol Chem 1967, 31:200-206.

12. Xiang L, Moore BS: Biochemical characterization of a prokaryotic phenylalanine ammonia lyase. J Bacterio/ 2005, 187:4286-4289.

13. Xu F, Cai R, Cheng S, Du H, Wang Y, Cheng S: Molecular cloning, characterization and expression of phenylalanine ammonia-lyase gene from Ginkgo biloba. Afr J Biotechnol 2008, 7:721-729.

14. Okada T, Mikage M, Sekita S: Molecular characterization of the phenylalanine Ammonia-Lyase from Ephedra sinica. Biol Pharm Bull 2008, 31:2194-2199.

15. Minami E, Ozeki Y, Matsuoka M, Koizuka N, Tanaka Y: Structure and some characterization of the gene for phenylalanine ammonialyase from rice plants. Eur J Biochem 1989, 185:19-25.

16. Lu BB, Du Z, Ding RX, Zhang L, Yu XJ, Liu CH, Chen WS: Cloning and characterization of a differentially expressed phenylalanine ammonia-lyase gene (liPAL) after genome duplication from tetraploid Isatis indigotica Fort. J Integr Plant Biol 2006, 48(12):1439-1449.

17. Wanner LA, Li G, Ware D, Somssich IE, Davis KR: The phenylalanine ammonia lyase gene family in Arabidopsis thaliana. Plant Mol Biol 1995, 27:327-338.

18. Gao JH, Zhang SW, Cai F, Zheng XJ, Lin N, Qin XB, Ou YC, Gu XP, Zhu XH, Xu Y, Chen $\mathrm{F}$ : Characterization, and expression profile of a phenylalanine ammonia lyase gene from Jatropha curcas L. Mol Biol Rep 2012, 39:3443-3452.

19. Jiang YM, Xia N, Li XD, Shen WB, Liang $\sqcup$, Wang CY, Wang R, Peng F, Xia B: Molecular cloning and characterization of a phenylalanine ammonia-lyase gene (LrPAL) from Lycoris radiate. Mol Biol Rep 2011, 38:1935-1940.

20. Ritter H, Schulz GE: Structural basis for the entrance into the phenylpropanoid metabolism catalyzed by phenylalanine ammonia-lyase. Plant Cell 2004, 16:3426-3436.

21. Joos HJ, Hahlbrock K: Phenylalanine ammonia-lyase in potato (Solanum tuberosumL.). Genomic complexity, structural comparison of two selected genes and modes of expression. Eur J Biochem 1992, 204:621-629.

22. Chang A, Lim MH, Lee SW, Robb EJ, Nazar RN: Tomato phenylalanine ammonia-lyase gene family, highly redundant but strongly underutilized. J Biol Chem 2008, 283:33591-33601 
23. Huang JL, Gu M, Lai ZB, Fan BF, Shi K, Zhou YH, Yu JQ, Chen ZX: Functional analysis of the arabidopsis PAL Gene family in plant growth, development, and response to environmental stress. Plant Physiol 2010, 153:1526-1538.

24. Schwede TF, Re'tey J, Schulz GE: Crystal structure of histidine ammonia-lyase revealing a novel polypeptide modification as the catalytic electrophile. Biochemistry 1999, 38:5355-5361.

25. Watanabe SK, Hernandez-Velazco G, Iturbe-Chinas F, Lopez-Munguia A: Phenylalanine ammonia lyase from Sporidiobolus pararoseus and Rhodosporidium toruloides: application for phenylalanine and tyrosine deamination. World J Microbiol Biotechnol 1992, 8:406-410.

26. Mukherjee PK, Mukherjee D, Maji AK, Rai S, Heinrich M: The sacred lotus (Nelumbo nucifera)-phytochemical and therapeutic profile. J Pharm Pharmacol 2009, 61(4):407-422.

27. Kashiwada $Y$, Aoshima A, Ikeshiro Y, Chen YP, Furukawa H, Itoigawa M, Fujioka T, Mihashi K, Cosentino LM, Morris-Natschke SL, Lee KH: Anti-HIV benzylisoquinoline alkaloids and flavonoids from the leaves of Nelumbo nucifera, and structure-activity correlations with related alkaloids. Bioorg Med Chem 2005, 13:443-448.

28. Hsu J: Late cretaceous and cenozoic vegetation in China, emphasizing their connections with north America. Ann Mo Bot Gard 1983, 70:490-508.

29. Ming R, Vanburen R, Liu Y, Yang M, Han Y, Li LT, Zhang Q, Kim MJ, Schatz MC, Campbell M, Li J, Bowers JE, Tang H, Lyons E, Ferguson AA, Narzisi G, Nelson DR, Blaby-Haas CE, Gschwend AR, Jiao Y, Der JP, Zeng F, Han J, Min XJ, Hudson KA, Singh R, Grennan AK, Karpowicz SJ, Watling JR, Ito K, et al: Genome of the long-living sacred lotus (Nelumbo nucifera Gaertn.). Genome Biol 2013, 14:R41.

30. Wang Y, Fan G, Liu Y, Sun F, Shi C, Liu X, Peng J, Chen W, Huang X, Cheng S, Liu Y, Liang X, Zhu H, Bian C, Zhong L, Lv T, Dong H, Liu W, Zhong X, Chen J, Quan Z, Wang Z, Tan B, Lin C, Mu F, Xu X, Ding Y, Guo AY, Wang J, Ke $W$ : The sacred lotus genome provides insights into the evolution of flowering plants. Plant J 2013, 76:557-567.

31. Schmidt K, Heberle B, Kurrasch J, Nehls R, Stahl DJ: Suppression of phenylalanine ammonia lyase expression in sugar beet by the fungal pathogen Cercospora beticola is mediated at the core promoter of the gene. Plant Mol Biol 2004, 55:835-852.

32. Angiosperm Phylogeny Group: An update of the Angiosperm Phylogeny Group classification for the orders and families of flowering plants: APG III. Bot J Linn Soc 2003, 141:399-436.

33. Rösler J, Krefel F, Amrhein N, Sohmid J: Maize phenylalanine ammonia-lyase activity. Plant Physiol 1997, 113:175-179.

34. Bagal UR, Leebens-Mack JH, Lorenz WW, Dean JFD: The phenylalanine ammonia lyase (PAL) gene family shows a gymnosperm-specific lineage. BMC Genomics 2012, 13(Suppl.3):S1.

35. Hsieh LS, Hsieh YL, Yeh CS, Cheng CY, Yang CC, Lee PD: Molecular characterization of a phenylalanine ammonia-lyase gene (BoPAL1) from B.oldhamii. Mol Biol Rep 2011, 38:283-290.

36. Tan RX, Zou WX: Endophytes: a rich source of functional metabolites. Nat Prod Rep 2001, 18:448-459.

37. Fiona CC, Laurence BD, Norman GL: The Arabidopsis phenylalanine ammonia lyase gene family: kinetic characterization of the four PAL isoforms. Phytochemistry 2004, 65:1557-1564.

38. Lee SW, Robb J, Nazar RN: Truncated phenylalanine ammonia-lyase expression in tomato (Lycopersicon esculentum). J Biol Chem 1992, 267:11824-11830

39. Chaw SM, Zhaekikh A, Sung HM, Lau TC, Li WH: Molecular phylogeny of extant gymnosperms and seed plant evolution: analysis of nuclear $18 \mathrm{~S}$ rRNA sequences. Mol Biol Evol 1997, 14:56-68.

40. Keeling PJ, Palmer JD: Horizontal gene transfer in eukaryotic evolution Nat Rev Genet 2008, 9:605-618.

41. Bergthorsson U, Adams KL, Thomason B, Palmer JD: Widespread horizontal transfer of mitochondrial genes in flowering plants. Nature 2003, 424:197-201.

42. Bergthorsson U, Richardson AO, Young GJ, Goertzen LR, Palmer JD: Massive horizontal transfer of mitochondrial genes from diverse land plant donors to the basal angiosperm Amborella. Proc Natl Acad Sci USA 2004, 101:17747-17752.

43. Hao W, Richardson AO, Zheng Y, Palmer JD: Gorgeous mosaic of mitochondrial genes created by horizontal transfer and gene conversion. Proc Natl Acad Sci USA 2010, 107:21576-21581.
44. Rumpho ME, Worful JM, Lee J, Kannan K, Tyler MS, Bhattacharya D Moustafa M, Manhart JR: Horizontal gene transfer of the algal nuclear gene psbO to the photosynthetic sea slug Elysia chlorotica. Proc Natl Acad Sci USA 2008, 105:17867-17871.

45. Yang J, Huang JX, Gu HY, Zhong Y, Yang ZH: Duplication and adaptive evolution of the chalcone synthase genes of Dendranthema (Asteraceae). Mol Biol Evol 2002, 19:1752-1759.

46. Kumar A, Ellis BE: The phenylalanine ammonia-lyase gene family in raspberry. Structure, expression, and evolution. Plant Physiol 2001, 127:230-239.

47. Röther D, Poppe L, Morlock G, Viergutz S, Rétey J: An active site homology model of phenylalanine ammonia-lyase from Petroselinum crispum. Eur J Biochem 2002, 269:3065-3075.

48. Allwood EG, Davies DR, Gerrish C, Ellis BE, Bolwell GP: Phosphorylation of phenylalanine ammonia-lyase: evidence for a novel protein kinase and identification of the phosphorylated residue. FEBS Lett 1999, 457:47-52

49. Xu EY, Moore FL, Reijo Pera RA: A gene family required for human germ cell development evolved from an ancient meiotic gene conserved in all metazoans. Proc Natl Acad Sci USA 2001, 98:7414-7419.

50. Li JK, Zhou EX, Li DX, Huang SQ: Multiple northern refugia for Asian sacred lotus, an aquatic plant with characteristics of ice-age endurance. Aust J Bot 2010, 58:463-472.

51. Thompson JD, Gibson TJ, Higgins DG: Multiple sequence alignment using ClustalW and ClustalX. Curr Protoc Bioinformatics 2002, 00:2.3.1-2.3.22.

52. Tamura K, Peterson D, Peterson N, Stecher G, Nei M, Kumar S: MEGA5: molecular evolutionary genetics analysis using likelihood, distance, and parsimony methods. Mol Biol Evol 2011, 28:2731-2739.

53. Guindon S, Delsuc F, Dufayard JF, Gascuel O: Estimating maximum likelihood phylogenies with PhyML. Methods Mol Biol 2009, 537:113-137.

54. Huelsenbeck JP, Ronquist F: MRBAYES: Bayesian inference of phylogenetic trees. Bioinformatics 2001, 17:754-755

55. Gambino G, Perrone I, Gribaudo I: A Rapid and effective method for RNA extraction from different tissues of grapevine and other woody plants. Phytochem Anal 2008, 19:520-525.

56. Bradford MM: A rapid and sensitive method for the quantitation of microgram quantities of protein utilizing the principle of protein-dye binding. Anal Biochem 1976, 72:248-254.

57. D'Cunha GB, Satyanarayan S, Nair PM: Purification of phenylalanine ammonia lyase from Rhodotorula glutinis. Phytochemistry 1996, 42:17-20.

doi:10.1186/1471-2148-14-100

Cite this article as: Wu et al:: Molecular evolution and functional characterisation of an ancient phenylalanine ammonia-lyase gene (NnPAL1) from Nelumbo nucifera: novel insight into the evolution of the PAL family in angiosperms. BMC Evolutionary Biology 2014 14:100.

\section{Submit your next manuscript to BioMed Central and take full advantage of:}

- Convenient online submission

- Thorough peer review

- No space constraints or color figure charges

- Immediate publication on acceptance

- Inclusion in PubMed, CAS, Scopus and Google Scholar

- Research which is freely available for redistribution 